\title{
Regionaler Nationalismus und Autonomie: ein Weg zur Konfliktlösung in westlichen Demokratien?
}

von Simon Schubert

Verschiedene westlich-demokratische Staaten (u.a. Belgien, das Vereinigte Königreich und Spanien) wurden in den vergangenen Jahrzehnten durch Nationalitätenkonflikte herausgefordert. Nationalistische Bewegungen stellen die territoriale Integrität in Frage, indem sie für bestimmte Regionen unabhängige staatliche Strukturen fordern. Um diese Konflikte zu entschärfen, wird häufig auf das Mittel begrenzter politischer Autonomie für die betreffenden Regionen zurückgegriffen. Es ist jedoch fraglich, inwiefern dadurch tatsächlich das nationalistische Separationsstreben und dessen Unterstützung durch die Bevölkerung reduziert werden können. Ein Vergleich der Konflikte im Baskenland, in Katalonien und in Flandern zeigt, dass auch sehr weitreichende Autonomielösungen weder dazu führen, dass nationalistische Akteure von ihren Unabhängigkeitsforderungen Abstand nehmen, noch dass sie signifikant an Unterstützung verlieren. Allenfalls reduziert sich die Konfliktintensität.

During the last decades, several Western democratic states (e.g. Belgium, the United Kingdom and Spain) have been challenged by nationalist conflicts. The respective movements question these countries' territorial integrity by claiming independent statehood for their regions. In order to mitigate these conflicts, limited political autonomy is usually granted. However, it remains questionable whether the nationalist aspirations for separation and their support among the population can be reduced this way. A comparative analysis of the conflicts in the Basque Country, Catalonia and Flanders shows that even far-reaching autonomy does neither cause nationalist actors to refrain from their claims to independence nor lead to a decline in popular support of nationalism. At best, the conflicts' forms become less intense.

\section{Einleitung}

Die jüngsten Schwierigkeiten bei der Regierungsbildung in Belgien haben dem international sonst eher wenig beachteten Konflikt zwischen den Bevölkerungsgruppen dieses Staates ein erhöhtes Maß an Aufmerksamkeit verschafft. Dies gilt auch für den damit einhergehenden Erfolg flämisch-separatistischer Parteien, 
insbesondere der Nieuw-Vlaamse Alliantie („Neu-flämische Allianz“ - N-VA), die bei der Parlamentswahl 2010 stärkste Kraft wurde. Regional-nationalistische ${ }^{1}$ Bewegungen, die die Schaffung eines neuen unabhängigen Staates anstreben, gibt es jedoch auch in anderen westeuropäischen Staaten, insbesondere in Spanien und dem Vereinigten Königreich. Sie versuchen ihre Ziele teils mit gewaltsamen Mitteln (etwa die baskische ETA), teils auf friedliche Weise zu erreichen. Diese Ziele stellen aber jeweils eine unmittelbare Bedrohung der territorialen Integrität des betroffenen Staates dar.

Da es sich bei den angesprochenen Ländern in Westeuropa um demokratische Rechtsstaaten handelt, können sie dieser Bedrohung nicht mit Repression begegnen. Stattdessen wurde und wird versucht, dem Separationsbestreben durch Gewährung politischer Autonomie ${ }^{2}$ innerhalb des Staatswesens zu begegnen. Von einer solchen begrenzten Selbstbestimmung erhofft man sich zumeist einen Rückgang des Verlangens nach Unabhängigkeit. Für dieses Vorgehen stehen als prominente Beispiele die Regionalisierung Spaniens im Rahmen des „Staates des Autonomen Gemeinschaften“, die Entwicklung Belgiens zum Föderalstaat und die britische devolution.

Die beiden erstgenannten Politiken laufen bereits seit mehreren Jahrzehnten. Insofern erscheint es angebracht, ihren Erfolg einer vergleichenden Untersuchung zu unterziehen. In diesem Beitrag wird daher der Frage nachgegangen, inwiefern die Gewährung politischer Autonomie ein geeignetes Mittel darstellt, um Konflikte mit regionalen Nationalismen in demokratischen Rechtsstaaten zu lösen oder zumindest zu akkommodieren. ${ }^{3}$

Hierfür werden die drei Fälle des baskischen und des katalanischen Nationalismus in Spanien sowie des flämischen Nationalismus in Belgien dahingehend geprüft, ob die Politik der Schaffung sog. Autonomer Gemeinschaften in Spanien bzw. die Föderalisierung in Belgien dazu führten, dass die Unterstützung des

1 Der Begriff „Nationalismus“ wird im Folgenden ausschließlich für Ideologien und Bewegungen gebraucht, deren Ziel es ist, staatliche und nationale Grenzen zur Deckung zu bringen (vgl. Hechter, M.: Containing Nationalism, Oxford, 2000, 7). Im Fall des regionalen Nationalismus bedeutet dies konkret das Streben nach der Schaffung eines unabhängigen Staates für eine (postulierte) Nation auf einem Teilgebiet (Region) eines bestehenden Staates.

2 Regionale politische Autonomie ist sowohl in Form einer bundesstaatlichen Organisationsform als auch in Form eines regionalisierten Einheitsstaates möglich.

3 Vgl. hierzu Schubert, S.: Regionaler Nationalismus und föderale Arrangements: Lösung oder Verschärfung des Konflikts?, Berlin, 2011. 
regionalen Nationalismus und das Bestreben nach Separation zurückgingen oder zumindest die Intensität der Konfliktaustragung reduziert wurde.

Die Fallauswahl bedarf in mehrerlei Hinsicht der Erklärung. So ist die Untersuchung von Fällen aus Belgien und Spanien nicht nur dadurch bedingt, dass in beiden Staaten seit den 1970er Jahren durch die Übertragung von Aufgaben und Entscheidungskompetenzen auf regionale Gebietskörperschaften versucht wurde, nationalistischen Forderungen entgegenzuwirken. Hinzukommt, dass in beiden Staaten ähnliche historische, staatsorganisatorische und sozio-ökonomische Voraussetzungen gegeben sind: Beide weisen eine nachhaltige Tradition des französisch geprägten zentralisierten Einheitsstaates auf, in beiden sind es gerade überdurchschnittlich wirtschaftsstarke und wohlhabende Regionen, die nach Selbständigkeit streben, und beide Staaten sind Mitglieder der Europäischen Union. Hingegen ist etwa der Fall Schottland in Großbritannien nicht nur durch die spezifisch angelsächsische Rechtstradition geprägt, sondern vor allem auch dadurch, dass Schottland trotz der seit 1707 bestehenden Union mit England in vielen staatlichen Bereichen, etwa im Rechts- und im Bildungswesen, ein erhebliches $\mathrm{Ma} ß$ an Eigenständigkeit bewahren konnte. Zudem ist Schottland innerhalb des Vereinigten Königreichs eine nicht nur geographisch, sondern auch ökonomisch eher periphere Region.

Innerhalb des spanischen Kontexts werden die Konflikte in Katalonien und dem Baskenland als eigenständige Fälle behandelt, da sie sowohl hinsichtlich der Voraussetzungen als auch der Konfliktintensität sowie in Art und Umfang der durch den Zentralstaat gewährten Autonomie erhebliche Unterschiede aufweisen. So ist der katalanische Nationalismus eine Bewegung, in der bürgerlich-liberale Kräfte und auch Teile der Arbeiterbewegung früh eine maßgebliche Rolle innehatten, während das baskische Gegenstück lange stark konservativ-klerikal geprägt war und sich auf eine soziale Basis von Industrialisierungsverlierern stützte. Im Baskenland war und ist der Konflikt besonders durch terroristische Gewalt geprägt - ein Phänomen, das in Katalonien vernachlässigbar ist. Zudem wurden dem Baskenland in den letzten Jahrzehnten deutlich weiterreichende autonome Befugnisse eingeräumt als Katalonien, insbesondere im Bereich der Finanzordnung.

Es mag schließlich verwundern, warum der in vieler Hinsicht bipolar strukturierte belgische Konflikt hier nur mit Blick auf den einen Pol, die flämische Seite, analysiert wird. Dies erklärt sich dadurch, dass es nur auf dieser Seite einen relevanten Nationalismus gab und gibt, der für eine flämische Nation einen unabhängigen Staat fordert. Auf der frankophonen Seite war zwar zeitweise die - 
inzwischen erfüllte - Forderung nach regionaler Autonomie in der Wirtschaftspolitik verbreitet. Akteure, die die Schaffung eines eigenen Staates oder die Verbindung der französischsprachigen Gebiete Belgiens mit Frankreich verlangten, blieben jedoch stets marginal. Inzwischen wenden sich die Vertreter der frankophonen Bevölkerung in den Verhandlungen um die künftige Struktur des belgischen Staates explizit gegen weitere Dezentralisierungsschritte.

\section{Nationalismus und regionale Autonomie}

Regional-nationalistische Forderungen beziehen sich zumeist auf einen klar abgegrenzten Teil des Staatsgebiets und verweisen auf dessen Besonderheiten sprachlicher, kultureller, historischer oder auch religiöser Art gegenüber dem Rest des Gesamtstaates. Die (originäre) Bevölkerung dieses Territoriums wird als Nation ${ }^{4}$ postuliert und daraus der Anspruch auf einen eigenen, souveränen Staat abgeleitet.

Seitens der Vertreter des Zentralstaates wird in aller Regel der Anspruch auf Unabhängigkeit abgelehnt, meist auch das Postulat der ,eigenen Nation“. In demokratischen Rechtsstaaten ist man jedoch häufig, ggf. auch nolens volens bereit, die Besonderheiten der fraglichen Region grundsätzlich anzuerkennen und daraus auch ein Recht auf beschränkte Selbstbestimmung abzuleiten. Forderungen nach regionaler Eigenständigkeit beispielsweise in Fragen der Kulturund Bildungspolitik werden meist als grundsätzlich legitim angesehen.

Eine Reform der Staatsorganisation mit dem Ziel begrenzter regionaler Autonomie wird aber nicht nur aus normativen Erwägungen verfolgt. Vielmehr wird damit auch das Ziel verfolgt, die Gefahr eines nationalistischen Maximalerfolgs in Form einer Separation zu reduzieren, indem die begrenzte regionale Autonomie als für beide Seiten akzeptabler Kompromiss ins Spiel gebracht wird. Damit verbindet sich die Hoffnung, dass die nationalistischen Akteure Abstand von ihrer Forderung nach Unabhängigkeit nehmen bzw. dass sie in dieser Hinsicht

4 Es kann inzwischen als Konsens in den Sozialwissenschaften gelten, dass Nationen nicht als primordialunveränderliche Einheiten verstanden werden können, sondern als sozial konstruierte, grundsätzlich veränderbare Gemeinschaften anzusehen sind (vgl. statt vieler Anderson, B.: Die Erfindung der Nation. Zur Karriere eines folgenreichen Konzepts, Frankfurt a.M./New York, 1996). Zwar lassen sich bis zu einem gewissen Grad empirische Indikatoren nutzen, um die (Nicht-)Existenz einer Nation zu belegen (vgl. Lepsius, M.R.: Die Teilung Deutschlands und die deutsche Nation, in: ders.: Demokratie in Deutschland. Soziologisch-historische Konstellationsanalysen, Göttingen, 1993, 196-228), doch sind Abgrenzungsfragen im Einzelfall kaum objektiv zu entscheiden, sondern vielmehr politischideologischer Natur. 
die Unterstützung der Bevölkerung verlieren, wenn ein erhebliches Maß an regionaler Selbstbestimmung auch ohne staatliche Trennung und die damit möglicherweise verbundenen negativen Konsequenzen zu erhalten ist. Diese Annahme ist auch in der Fachliteratur weit verbreitet. Auf den Punkt gebracht hat sie Juan J. Linz mit seiner Aussage, ,democratic federalism can contribute successfully to solve the conflict between national, linguistic and religious groups within a state and prevent its disintegration. ${ }^{\circ 5}$

Auch wenn diese Annahme auf den ersten Blick sehr einleuchtend sein mag, lässt sie sich doch hinterfragen. So ist keineswegs gesagt, warum Vertreter einer nationalistisch-separatistischen Ideologie sich mit einer nur partiellen Erfüllung ihrer Forderung zufrieden geben und sie diese nicht vielmehr als ersten Schritt auf dem Weg zur angestrebten Unabhängigkeit begreifen sollten. Vorausgesetzt, dass es ihnen gelingt, in der autonomen Gebietskörperschaft maßgebliche politische Ämter, insbesondere die regionale Exekutive, zu besetzen, stehen ihnen zudem dadurch bedeutende Ressourcen zur Verfügung, die sie fortan für ihre Zwecke einsetzen können. Dazu gehören insbesondere die örtliche öffentliche Verwaltung, ggf. einschließlich bewaffneter Sicherheitskräfte in Form einer eigenständigen regionalen Polizei, aber auch in der Regel das Bildungswesen. Dies ist insofern bedeutsam, als es dafür genutzt werden kann, das Nationalbewusstsein (bezogen auf die postulierte regionale Nation) in der Bevölkerung langfristig zu festigen und zu stärken. ${ }^{6}$ Insofern lässt sich mit Richard Simeon von einer „Janus-faced quality of federalism“" sprechen:

„It is once a vehicle for accommodation, a way of reconciling the minority nations and groups to the larger whole, and a device for perpetuating and institutionalizing the very cleavages it is designed to manage, which may provide the institutional resources from which to launch a successful secession movement." ${ }^{\text {"7 }}$

5 Linz, J.J.: Democracy, Multinationalism and Federalism, in: Merkel, W./Busch, A. (Hg.): Demokratie in Ost und West. Für Klaus von Beyme, Frankfurt a.M., 1999, 382-401, hier 387. Vgl. auch Amoretti, U.M.: Introduction: Federalism and Territorial Cleavages, in: ders./Bermeo, N. (Hg.): Federalism and Territorial Cleavages, Baltimore/London, 2004, 1-23.

6 Vgl. Martiniello, M.: The National Question and the Political Construction of Immigrant Ethnic Communities in Belgium, in: Hargreaves, A.G./Leaman, J. (Hg.): Racism, Ethnicity and Politics in Contemporary Europe, Aldershot/Brookfield, VT, 1995, 131-144, hier 135.

7 Simeon, R.: Canada: Federalism, Language, and Regional Conflict, in: Amoretti, U.M./Bermeo, N. (Hg.): Federalism and Territorial Cleavages, Baltimore/London, 2004, 93-122, hier 93. 


\section{Historische, rechtliche und politische Voraussetzungen}

\section{Späte Integration und frühe Gegenreaktionen: das Baskenland und Spanien}

Die Eigenheit des Baskenlandes basiert vor allem auf der baskischen Sprache. ${ }^{8}$ Über ihre Ursprünge und mögliche Verwandtschaften zu anderen Sprachen liegen trotz intensiver Forschung keine gesicherten Erkenntnisse vor. Sie wird jedenfalls seit mehreren Jahrtausenden im westlichen Pyrenäenraum gesprochen und hat sich somit als einzige Sprache aus vor-indogermanischer Zeit in Mittelund Westeuropa erhalten.

Die im heutigen Spanien gelegenen baskischen Gebiete kamen zwischen dem 11. und dem frühen 16. Jahrhundert unter die Oberhoheit der kastilischen bzw. spanischen Krone. Bis ins 19. Jahrhundert unterlagen sie jedoch mit dem System der sog. fueros einem rechtlich-politischen Sonderstatus. ${ }^{9}$ Dieser beinhaltete insbesondere eine Befreiung von Zöllen und Militärdienst sowie umfangreiche lokale Selbstverwaltungsrechte und eine Beibehaltung des tradierten gewohnheitsrechtlichen Privatrechts. Die - insbesondere auch vom aufstrebenden baskischen Großbürgertum betriebene - weitgehende Abschaffung dieses Systems 1876 führte, gepaart mit gesellschaftlichen Veränderungen, zu einem hohen Maß an Verunsicherung bei den Verlierern von Modernisierung und Industrialisierung, vor allem der Landbevölkerung und dem traditionellen Kleinbürgertum, die den Nährboden für den baskischen Nationalismus bildete. ${ }^{10}$

Dieser entstand in seiner modernen Form ab den 1880er Jahren unter maßgeblicher Federführung von Sabino Arana, der 1894 die noch heute bestehende Baskische Nationalistische Partei (PNV) gründete. ${ }^{11}$ Das Ziel war von Anfang an ein unabhängiger, katholisch geprägter baskischer Staat. Entsprechend wurden weite Teile des Klerus auch früh zu einem wesentlichen Träger der nationalistischen Ideologie. Mit der Ausrufung der spanischen Republik 1931 verbanden sich von baskischer Seite Hoffnungen auf ein Autonomiestatut, das jedoch erst nach Aus-

8 Vgl. ausführlich zum Folgenden: Kasper, M.: Baskische Geschichte in Grundzügen, Darmstadt, 1997.

9 Conversi, D.: The Basques, the Catalans and Spain. Alternative Routes to Nationalist Mobilisation, London, 1997, 45ff.; Diez Medrano, J.: Divided Nations. Class, Politics, and Nationalism in the Basque Country and Catalonia, Ithaca, NY/London, 1995, 23ff.

10 Waldmann, P.: Militanter Nationalismus im Baskenland, Frankfurt a.M., 1990, $29 \mathrm{ff}$.

11 Diez Medrano, J., a.a.O., $75 \mathrm{ff}$. 
bruch des Bürgerkriegs 1936 gewährt wurde und mit der Eroberung der baskischen Gebiete durch die Truppen General Francos 1937 obsolet wurde.

Die anschließende, bis 1975 währende Diktatur Francos war im Baskenland (wie auch in Katalonien, s.u.) besonders repressiv. Der öffentliche Gebrauch der baskischen Sprache war ebenso untersagt wie jede Form der politischen Betätigung, die von der offiziellen Linie des einen, homogenen Spanien abwich. Unter diesen Bedingungen kam es 1959 zu einer radikalen Abspaltung vom PNV unter dem Namen ETA (Euskadi ta Askatasuna - „Baskenland und Freiheit“), die sich von den katholisch-konservativen Wurzeln des Nationalismus entfernte, deutlich nach links rückte und Inspiration bei den sog. Befreiungsbewegungen in der Dritten Welt suchte. ${ }^{12}$ Angehörige von ETA waren erstmals 1968 in eine bewaffnete Auseinandersetzung mit der Polizei verwickelt; bald darauf begann die Organisation mit gezielten Anschlägen auf Repräsentanten des Regimes, deren erster Höhepunkt 1973 die Ermordung des Regierungschefs und prospektiven Franco-Nachfolgers Carrero Blanco war. Als der Diktator 1975 starb und die Demokratisierung Spaniens, und damit einhergehend, die Einrichtung einer dauerhaften Autonomie für das Baskenland folgen sollte, bestanden somit zwei Hauptströmungen des Nationalismus, die zwar beide das Ziel der Unabhängigkeit verfolgten, sich ansonsten aber stark unterschieden: der gewaltfrei agierende, katholisch-konservative PNV einerseits und die zur Terrororganisation gewandelte ETA andererseits, die sich mit ihrem diffus-sozialistischen Weltbild ebenfalls auf eine breite Massenbasis stützen konnte.

Staatsorganisatorisch-administrativ war Spanien im 19. Jahrhundert zum zentralisierten Einheitsstaat geworden, der dem Vorbild des französischen Nachbarn folgte, etwa in der Einteilung des Staatsgebiets in 50 Provinzen, die strukturell und funktional den Départements entsprachen. Für die vier baskischen Provinzen bestanden auch nach der Abschaffung der fueros 1876 jedoch Sonderregelungen hinsichtlich der Finanzordnung: Die Steuererhebung oblag der Selbstverwaltung der Provinzen, die einen Pauschalbetrag an den Zentralstaat abführten. Dieses System war von Franco für die beiden Provinzen, die die republikanische Regierung unterstützt hatten, 1937 abgeschafft worden - in Navarra und Álava, die Franco unterstützt hatten, blieb es bestehen.

12 Diez Medrano, J., a.a.O., 136ff.; Mees, L.: Nationalism, Violence and Democracy. The Basque Clash of Identities, Basingstoke, 2003, 23ff.; Watson, C.J.: Basque Nationalism and Political Violence. The Ideological and Intellectual Origins of ETA, Reno, 2007. 


\section{Katalonien: kulturelle „Wiedergeburt“ und Nationalismus}

Auch die katalanische Kultur definiert sich vor allem über die eigene Sprache, die - wie das kastilische Spanisch - aus dem Vulgärlatein hervorgegangen ist. ${ }^{13}$ Die Tradition Kataloniens als eigenständiges Gemeinwesen lässt sich auf die aus dem Fränkischen Reich hervorgegangene Grafschaft Barcelona zurückführen. Im späten Mittelalter dominierte Katalonien das aragonesische Reich, das seinerzeit zu den bedeutenden Mächten im Mittelmeerraum zählte. Der wirtschaftliche Aufschwung ermöglichte eine kulturelle Blüte. Seit Ende des 15. Jahrhunderts zunächst in Personalunion mit dem Königreich Kastilien verbunden, verlor Katalonien nach dem Sieg der Bourbonen im Spanischen Erbfolgekrieg 1714 alle bis dahin noch bestehenden Sonder- und Selbstverwaltungsrechte und wurde voll in den sich herausbildenden Einheitsstaat integriert. Die katalanische Sprache konnte sich zwar erhalten, sank aber auf die Rolle einer Umgangssprache der einfachen Bevölkerung herab, während das kastilische Spanisch alleinige Amts-, Kultur- und Bildungssprache wurde.

Der moderne katalanische Nationalismus hatte seine Wurzeln zunächst in einer seit Mitte des 19. Jahrhunderts feststellbaren Rückbesinnung auf die eigenen sprachlichen und kulturellen Traditionen, der sog. Renaixença (,Wiedergeburt"). ${ }^{14}$ Diese verband sich bald mit Forderungen nach politischer Selbstbestimmung, die von breiten Bevölkerungsschichten getragen wurden - so waren die aufstrebenden katalanischen Industriellen zunehmend unzufrieden damit, dass ihre Bedürfnisse und wirtschaftspolitischen Forderungen von den Regierungen in Madrid ignoriert wurden. Entsprechend war der katalanische Nationalismus von Anfang an eine heterogene Bewegung, die konservative und liberale Akteure umfasste und früh für Teile der Arbeiterbewegung anschlussfähig wurde. ${ }^{15}$ Die Forderungen reichten von begrenzter politischer und ökonomischer Selbstbestimmung bis hin zu voller staatlicher Unabhängigkeit. Es konnte sich auch keine einzelne Organisation über längere Zeit als alleinige Repräsentation des katalanischen Nationalismus und Regionalismus etablieren, so dass immer mehrere Vereinigungen bzw. Parteien nebeneinander bestanden.

13 Vgl. zum Folgenden McRoberts, K.: Catalonia. Nation Building without a State, Oxford, 2001 sowie Guibernau, M.: Catalan Nationalism. Francoism, Transition and Democracy, London/New York, 2004.

14 Conversi, D.: The Basques, the Catalans and Spain. Alternative Routes in Nationalist Mobilisation, London, 1997, 13ff.

15 Diez Medrano, J., a.a.O., 97ff. 
Innerhalb des zentralisierten spanischen Einheitsstaates wurde erstmals 1914 eine, wenngleich sehr beschränkte Form regionaler Selbstverwaltung für Katalonien gewährt; sie wurde 1925 wieder aufgehoben. Wesentlich weiterreichend war die von der republikanischen Regierung 1932 gewährte Autonomie Kataloniens, die allerdings bereits von 1934 bis 1936 wieder suspendiert wurde und schließlich mit dem Ende des Bürgerkriegs 1939 von Diktator Franco endgültig aufgehoben wurde.

Wie das Baskenland war auch Katalonien in der Folgezeit sowohl von besonders harter Repression betroffen als auch zunehmend ein Zentrum des Widerstands gegen das Regime, der hier allerdings auf gewaltfreie Aktionen zivilen Ungehorsams beschränkt blieb, die auch meist von kleinen, nur lose organisierten Gruppen getragen wurden. ${ }^{16}$

\section{Vom sozialen zum territorialen Konflikt: der belgische Sprachenstreit}

Der heutige belgische Staat entstand 1830 durch Abspaltung vom Vereinigten Königreich der Niederlande, einem erst 15 Jahre zuvor vom Wiener Kongress geschaffenen Staatsgebilde. ${ }^{17}$ Die politische Trennung des niederländischen Raumes in Norden (die heutigen Niederlande) und Süden (die heutigen Staaten Belgien und Luxemburg) ist jedoch wesentlich älter. Die auch heute noch bestehende Grenze zwischen Belgien und den Niederlanden entspricht im Wesentlichen der im Westfälischen Frieden 1648 festgelegten Linie. Nördlich davon befanden sich die Provinzen, die sich erfolgreich die Unabhängigkeit von der spanischen Herrschaft erkämpft hatten. Die südlichen Gebiete hingegen blieben zunächst bei Spanien, kamen 1714 unter österreichische Herrschaft und wurden 1794 von Frankreich annektiert. Bedeutsam ist dabei, dass die politische Grenze nicht der romanisch-germanischen Sprachgrenze entspricht. Diese verläuft, seit dem Ende der Völkerwanderung weitgehend unverändert, weiter südlich mitten durch das heutige Belgien.

In den unabhängigen Niederlanden entwickelte sich aus den örtlichen niederfränkischen Dialekten die moderne niederländische Standardsprache. Hingegen setze sich im Süden schon unter spanischer und österreichischer Herrschaft das

16 Vgl. Guibernau, M., a.a.O., 50ff.

17 Vgl. zum Folgenden ausführlich Erbe, M.: Belgien, Niederlande, Luxemburg. Geschichte des niederländischen Raumes, Stuttgart u.a., 1993 sowie Mabille, X.: Histoire politique de la Belgique. Facteurs et acteurs de changement, 4. Aufl., Brüssel, 2000. 
Französische als Amts- und Bildungssprache durch. Das heutige Flandern ${ }^{18}$ entspricht somit der Region, die zwar nördlich der romanisch-germanischen Sprachgrenze, aber südlich der politischen Grenze zwischen nördlichen und südlichen Niederlanden lag. In diesem Gebiet blieben die örtlichen, mit dem Niederländischen eng verwandten Mundarten bis ins 19. Jahrhundert auf die Funktion einer Umgangssprache beschränkt. Die staatliche Verwaltung, das Gerichts- und das höhere Bildungswesen und das kulturelle Leben spielten sich auf Französisch ab, dessen Beherrschung für den sozialen Aufstieg unumgänglich war.

Die von den Großmächten bewirkte erneute politische Vereinigung des niederländischen Raumes 1815 war nur von kurzer Dauer. Der neue Staat wurde von den Eliten des Nordens dominiert. Im Süden, d.h. dem heutigen Belgien, widersetze sich das Bürgertum massiv den Versuchen der Staatsführung, die Niederländische Sprache zur Amtssprache zu machen. Zudem war der Norden seit dem 16. Jahrhundert protestantisch geprägt, während der Süden katholisch geblieben war. Hinzu kam schließlich der autoritäre Regierungsstil des niederländischen Königshauses, der im stark von der Französischen Revolution beeinflussten Süden besonders auf Widerstand stieß. Aus diesen Faktoren speiste sich der Aufstand des Südens 1830, der zur Loslösung entlang der alten Grenze von 1648 und zur Schaffung eines neuen unabhängigen Staates führte - den Namen „Belgien" wählte man unter Rückgriff auf eine alte römische Provinzbezeichnung.

Dieser neue Staat war nach französischem Vorbild unitarisch-zentralistisch aufgebaut, die 1794 geschaffenen Départements lebten als Provinzen fort. Zur alleinigen Amtssprache wurde ebenfalls das Französische bestimmt, ungeachtet der Tatsache, dass mit den Bewohnern des nördlichen Landesteils mehr als die Hälfte der Bevölkerung das Niederländische (bzw. mit diesem eng verwandte Dialekte) als Muttersprache hatte. Damit war ein neuer Konflikt vorgezeichnet.

Unter diesen Voraussetzungen entstand um die Mitte des 19. Jahrhunderts die „Flämische Bewegung“, die zunächst vor allem kulturell geprägt war, aber schon bald auf die Gleichberechtigung der flämischen/niederländischen Sprache zielte. ${ }^{19}$ Dies richtete sich zunächst weniger gegen den französischsprachigen Süden

18 Seit dem späten 19. Jahrhundert bezeichnet der Terminus „Flandern“ den niederländischsprachigen Teil Belgiens. Dieser ist territorial nicht identisch mit der bis zum Ende des Ancien Régime bestehenden Grafschaft Flandern.

19 De Wever, B.: Die Flämische Bewegung. Geschichte und Geschichtsschreibung, in: Koll, J. (Hg.): Nationale Bewegungen in Belgien, Münster u.a., 2005, 73-107. 
des Landes, sondern gegen die eigenen Eliten im Norden, die auf dem Vorrang der Französischen Sprache beharrten. Der Kampf um die Gleichberechtigung des Niederländischen ging somit einher mit der zunehmenden Demokratisierung von Staat und Gesellschaft. Ein wesentlicher Erfolg konnte 1898 verzeichnet werden, als beide Sprachen in Gesetzgebung und Verwaltung de jure gleichgestellt wurden. Die faktische Durchsetzung der Zweisprachigkeit stieß jedoch im ausschließlich französischsprachigen Süden des Landes auf massive Widerstände, was wiederum die flämische Gegenreaktion hervorrief, im Norden das Französische noch weiter zurückzudrängen. ${ }^{20}$

Hieraus entwickelte sich das erstmals 1932 in Gesetzesform gegossene Prinzip der „territorialen Einsprachigkeit“. Demnach wurde das Land in festgelegte Sprachgebiete eingeteilt, in denen jeweils nur eine Sprache für den amtlichen Gebrauch und das öffentliche Bildungswesen zugelassen war. Flandern im Norden war fortan einsprachig Niederländisch, Wallonien im Süden einsprachig Französisch. Der Großraum Brüssel behielt einen zweisprachigen Status, ${ }^{21}$ hinzu kam schließlich das kleine deutsche Sprachgebiet an der Ostgrenze des Landes. Anfangs wurde die Sprachgrenze noch mithilfe regelmäßiger Volkszählungen der tatsächlichen Bevölkerungsentwicklung angepasst. Dies führte jedoch insbesondere zu eine Vergrößerung des zweisprachigen Brüsseler Gebiets zulasten Flanderns und rief zunehmenden flämischen Widerstand hervor, so dass die Sprachgrenze 1962 unveränderlich festgelegt wurde.

Durch diese konsequente Territorialisierung der Sprachpolitik wurde Belgien mithin in klar abgegrenzte, sprachlich definierte Gebietseinheiten unterteilt, in denen sich zunehmend auch ein regionales Bewusstsein herausbildete, das in Flandern zu einem nationalen Bewusstsein wurde. ${ }^{22}$ War das ursprüngliche Ziel der Flämischen Bewegung sprachliche Gleichstellung innerhalb Belgiens gewesen, so berief man sich jetzt auf eine flämische Nation, für die man politische

20 Delforge, P.: Ein Jahrhundert Wallonische Bewegung (1880-1980), in: Koll, J. (Hg.): Nationale Bewegungen in Belgien, Münster u.a., 2005, 109-133; Kesteloot, C.: The Growth of the Walloon Movement, in: Deprez, K/Vos, L (Hg.): Nationalism in Belgium. Shifting Identities, 1780-1995, Basingstoke u.a., 1998, 139-152.

21 In Brüssel, das auf ursprünglich flämischsprachigem Gebiet liegt, war - bedingt durch die geographische Nähe zur Sprachgrenze und die Hauptstadtfunktion - der Einfluss der französischen Sprache wesentlich intensiver als im übrigen Flandern, so dass spätestens seit Beginn des 20. Jahrhunderts die Große Mehrheit der Einwohner französischsprachig war und ist.

22 Maddens, B. u.a.: The National Consciousness of the Flemings and the Walloons. An Empirical Investigation, in: Deprez, K/Vos, L (Hg.): Nationalism in Belgium. Shifting Identities, 1780-1995, Basingstoke u.a., 1998, 198-208. Vgl. auch Billiet, J. u.a.: Does Belgium (Still) Exist? Differences in Political Culture between Flemings and Walloons, in: West European Politics, $29 / 5$ (2006), 912-932. 
Selbstbestimmungsrechte forderte. Zunehmend fand auch die Forderung nach einem unabhängigen flämischen Staat Gehör. Dennoch war die belgische Staatsorganisation bis in die 1960er Jahre weiterhin unitarisch-zentralisiert. Mit der Schaffung der Sprachgebiete ging zunächst keinerlei politische Dezentralisierung einher.

\section{Regionale Autonomie und ihre Auswirkung auf das Sezessions- streben}

Die drei hier untersuchten Fälle befanden sich Ende der 1960er (Flandern) bzw. Ende der 1970er Jahre (Katalonien, Baskenland) in ähnlichen Situationen: Die Staatsorganisation war unitarisch-zentralisiert und wurde von einer starken regional-nationalistischen Bewegung herausgefordert. Im Baskenland griffen Teile der Bewegung zu bewaffneter Gewalt, in allen drei Fällen konnte sie sich auf eine breite Massenbasis stützen und war ein unter demokratischen Bedingungen nicht mehr vernachlässigbarer Faktor. Die Reaktion des Zentralstaates war jeweils eine Reform der Staatsorganisation, durch die regionale Gebietskörperschaften eingerichtet und mit substanzieller politischer Autonomie ausgestattet wurden.

\section{Autonomie und Terror: Die Entwicklung des Baskenlandes}

Mit dem Tod des Diktators Franco 1975 war der Weg zur Demokratisierung Spaniens frei. Diese wurde unter dem neuen Staatsoberhaupt König Juan Carlos zügig in Angriff genommen, wobei man allerdings auf einen „sanften“ Übergang unter Beteiligung der reformbereiten Kräfte aus dem alten Regime setzte. Zugleich mussten die bisherigen Oppositionskräfte integriert werden. Landesweit waren das vor allem die Parteien der Linken, im Baskenland (wie auch in Katalonien) aber auch die Nationalisten. Ein erstes umfangreiches Reformgesetz wurde 1976 verabschiedet, 1977 fanden die ersten freien Wahlen seit 1936 statt, 1978 trat die bis heute fast unverändert gültige demokratische Verfassung in Kraft. Diese hielt formal an der unitarischen Staatsstruktur fest, eröffnete jedoch die Möglichkeit der Einrichtung sog. Autonomer Gemeinschaften, denen substanzielle Kompetenzen übertragen werden konnten. ${ }^{23}$

23 Vgl. ausführlich Wendland, K.: Spanien auf dem Weg zum Bundesstaat? Entstehung und Entwicklung der Autonomen Gemeinschaften, Baden-Baden, 1998. 
In den drei baskischen Kernprovinzen ${ }^{24}$ erzielten die verschiedenen nationalistischen Parteien bei den Wahlen 1977 ca. $36 \%$ der Stimmen. ${ }^{25}$ Schon dies allein hätte sie zum relevanten Akteur im damaligen Demokratisierungsprozess gemacht. Hinzu kam die breite Massenmobilisierung im nationalistischen Spektrum, die intensivierte Terroraktivität von ETA sowie schließlich die Tatsache, dass die Parteien der Linken, insbesondere die Sozialisten, die auf gesamtstaatlicher Ebene die größte Oppositionspartei bildeten, der Forderung nach baskischer Autonomie grundsätzlich befürworteten. ${ }^{26}$

In einer Phase des politischen Umbruchs im Zeichen der Demokratisierung stand der spanische Staat mithin vor der Alternative, entweder den nationalistischen Forderungen zumindest teilweise entgegenzukommen oder aber eine Eskalation in Kauf zu nehmen, die den gesamten Demokratisierungsprozess hätte gefährden können. Entsprechend wurden bereits Anfang 1978 - noch vor Inkrafttreten der Verfassung - vorläufige autonome Institutionen eingerichtet, die allerdings, mangels substanzieller Kompetenzen, eher symbolischen Charakter hatten. Parallel wurde in Allparteienverhandlungen ein Autonomiestatut ausgearbeitet, das nach Bestätigung durch das gesamtstaatliche Parlament sowie in Volksabstimmungen in den betroffenen Provinzen Ende 1979 in Kraft trat. ${ }^{27}$

Durch dieses Statut wurden im Baskenland ein autonomes Parlament und eine diesem verantwortliche Regierung eingerichtet. Diese Institutionen erhielten umfangreiche Kompetenzen in weiten Bereichen der Innen-, Wirtschafts-, Kultur-, Bildungs- und Sozialpolitik. Hervorzuheben ist die eigene baskische Polizei, die die Sicherheitskräfte des Zentralstaates weitgehend ersetzte, sowie die wiederhergestellten fiskalischen Sonderrechte für die Region. Demnach liegen die direkten Steuern vollständig in der Kompetenz der autonomen Institutionen. Diese handeln mit dem Zentralstaat einen Pauschalbetrag aus, der in dessen Haushalt abgeführt ist und der Finanzierung derjenigen öffentlichen Aufgaben dient, die der Zentralstaat im bzw. für das Baskenland noch wahrnimmt. Dieses System ist für das Baskenland - eine der wohlhabendsten Regionen Spaniens -

24 Álava, Guipúzcoa und Vizcaya, die heute die Autonome Gemeinschaft Baskenland bilden. Die baskischen Nationalisten beanspruchen darüber hinaus noch Gebiete auf französischer Seite sowie die spanische Provinz Navarra. In der letztgenannten ist jedoch nur ein kleinerer Teil der Bevölkerung baskischsprachig; Nationalisten konnten dort keine Mehrheiten erzielen. Die Provinz bildet heute eine eigenständige Autonome Gemeinschaft in Spanien.

25 Wahlergebnisse aus dem Baskenland im Folgenden nach Gobierno Vasco, Departamento de Interior: Archivo de Resultados Electorales, http://www9.euskadi.net.

26 Mees, L., a.a.O., 37.

27 Mees, L., a.a.O., 39ff.; Kasper, M., a.a.O., 187f. 
außerordentlich vorteilhaft, da es so aus dem Steuerverbund und dem interregionalen Finanzausgleich herausgelöst ist und nicht zur Finanzierung der unterdurchschnittlich entwickelten Landesteile herangezogen wird. Zudem wurde das Baskische gleichberechtigte Amts- und Unterrichtssprache.

Die Reaktion der baskischen Nationalisten auf diese Autonomieregelung war von Anfang an ambivalent. Die Vertreter der gemäßigten Linie um den PNV begrüßten einerseits die Möglichkeiten der Selbstregierung, machten aber zugleich deutlich, dass sie ihre Forderungen nach staatlicher Souveränität weiter aufrecht erhalten würden. ${ }^{28}$ Die Radikalen um ETA hingegen lehnten jede Lösung unterhalb der staatlichen Unabhängigkeit grundsätzlich ab. Verhandlungen über ein Autonomiestatut kamen für sie schon deshalb nicht in Frage, weil sie eine zumindest faktische Anerkennung der spanischen Souveränität implizierten. Die Zeit der Ausarbeitung und Einführung des Autonomiestatuts war daher auch von besonders intensiven Terroraktivitäten ETAs gekennzeichnet. ${ }^{29}$

Das Statut von 1979 bildet die bis heute unveränderte Rechtsgrundlage der Selbstregierung des Baskenlandes. Sie erwies sich bis Ende der 1990er Jahre als recht stabil, zumal die autonome Regierung meist von einer Koalition aus gemäBigten Nationalisten (PNV) und Sozialisten gebildet wurde und so ein Interessenausgleich zwischen nationalistischem und nicht-nationalistischem Lager möglich war. ${ }^{30}$ ETA und ihre Unterstützer blieben politisch marginalisiert, auch PNV-Politiker unterstützen den Einsatz aller rechtsstaatlichen Mittel gegen die Terrororganisation, der jedoch weiterhin jährlich eine zweistellige Zahl von Menschen zum Opfer fiel.

Dies änderte sich, als ab 1998 die PNV-Führung das Ziel der Selbständigkeit wieder priorisierte. Flankiert von einem - bald wieder gebrochenen - ETA„Waffenstillstand“ gab die Partei ihre bisherige Politik der Ausgrenzung der ETA-nahen Partei EH auf und suchte mit dieser die Verständigung über einen „Selbstbestimmungs“- bzw. Unabhängigkeitsplan, was zum Bruch des Bündnisses mit den Sozialisten führte. Fortan bildete der PNV Minderheitsregierungen, die von den radikalen Nationalisten toleriert wurden. ${ }^{31}$ 
Höhepunkte dieser Politik war 2003 die Vorstellung eines Entwurfs für ein ,politisches Statut", das das Autonomiestatut ersetzen sollte und nur noch eine unspezifizierte „freie Assoziation“ des Baskenlandes mit Spanien vorsah, - es wurde vom baskischen Parlament mit nationalistischer Mehrheit angenommen, von zentralstaatlichen Parlament aber mit großer Mehrheit zurückgewiesen $-{ }^{32}$ sowie 2008 das Vorhaben einer „Volksbefragung“ im Baskenland, die die autonome Regierung legitimieren sollte, auf dem Verhandlungsweg die Unabhängigkeit zu erlangen - sie wurde vom Verfassungsgericht untersagt. ${ }^{33}$ Vor offenem Rechtsbruch scheuten die gemäßigten Nationalisten jedoch zurück. 2009 kam es unter Führung der Sozialisten erstmals seit Bestehen der Autonomie zu einer Regionalregierung ohne nationalistische Parteien. Die dafür notwendige parlamentarische Mehrheit kam jedoch nur dadurch zustande, dass mithilfe eines neuen vom zentralstaatlichen Parlament erlassenen Parteiengesetzes alle radikalnationalistischen Kandidaturen aufgrund ihrer fehlenden Distanzierung vom ETA-Terror verboten worden waren. Die entsprechenden Organisationen riefen daraufhin zum Wahlboykott auf, der von ihren Anhängern auch weitgehend befolgt wurde.

Will man nun die Auswirkungen der spanischen Autonomie-Politik auf den Konflikt mit dem baskischen Nationalismus analysieren, so lässt sich zunächst feststellen, dass die wichtigsten Akteure des baskischen Nationalismus von ihrer Forderung nach staatlicher Unabhängigkeit nicht abgerückt sind. Dies betrifft sowohl die vergleichsweise gemäßigte langjährige Regierungspartei PNV als auch den radikaleren Flügel um die Terrororganisation ETA und die ihr nahestehenden Parteien, die in den vergangenen Jahren mehrfach Neugründungen und Umbenennungen vollzogen haben, um einem letztlich doch erfolgten Verbot zu entgehen. Der PNV hat sich zwar mit dem Autonomiestatut als „Zwischenlösung" arrangiert, aber in seiner Rhetorik das Ziel einer staatlichen Unabhängigkeit stets betont. Die radikaleren Parteien verhielten sich zu den durch das Autonomiestatut geschaffenen Institutionen ambivalent - die Handlungsformen reichten von Boykott bis zur Tolerierung von PNV-Minderheitsregierungen.

Ebenfalls weitgehend konstant blieb die Unterstützung des Nationalismus in der baskischen Bevölkerung - gemessen an den Stimmanteilen der nationalistischen Parteien. Seit den ersten Wahlen zum baskischen Parlament kommen diese stets auf Wahlergebnisse von insgesamt über $50 \%$. Die einzige Ausnahme hierzu 
bildete die Wahl 2009, bei der wie erwähnt aufgrund des Verbots aller radikalnationalistischen Wahlvorschläge eine nicht-nationalistische Mehrheit zustande kam.

Die Gewährung einer im internationalen Vergleich - auch und gerade mit Föderalstaaten - ausgesprochen weitreichenden Autonomie innerhalb des spanischen Staates hat also weder dazu geführt, dass die organisierten Vertreter der nationalistischen Ideologie von ihrer Forderung nach staatlicher Unabhängigkeit Abstand genommen hätten, noch ist ihre Unterstützung in der Bevölkerung signifikant zurückgegangen.

Zurückgegangen ist jedoch die Intensität des nationalistischen Terrorismus: Verantwortete ETA in den 1980er Jahren noch jedes Jahr eine zweistellige Zahl von Todesopfern, gingen die Anschläge seit Beginn der 1990er Jahre spürbar zurück und sind in den letzten Jahren nahezu zum Erliegen gekommen - zuletzt kam es im Juli 2009 zu einem ETA-Anschlag mit zwei Todesopfern. Diese „Pazifierung" des Konflikts ist in Teilen auf erfolgreiche Polizeiarbeit zurückzuführen, in Teilen aber auch auf einen signifikanten Rückgang der Unterstützung des Terrorismus in der baskischen Bevölkerung, der sich wiederum mit der politischen Autonomie erklären lässt.

\section{Regionalismus und Nationalismus: der Konflikt in Katalonien}

Wie im baskischen Fall verlief auch in Katalonien der Prozess der politischen Dezentralisierung Ende der 1970er Jahre zunächst parallel zur Demokratisierung Spaniens. ${ }^{34}$ Auch in Katalonien waren die nationalistischen Kräfte so stark, dass man sie im Rahmen eines den Namen verdienenden Demokratisierungsprozesses nicht ignorieren konnte. Allerdings waren die ideologischen Abgrenzungslinien deutlich weniger scharf als im Baskenland. Dies lag zum einen daran, dass auch innerhalb der nationalistischen Organisationen sowohl nationalistischseparatistische Tendenzen als auch moderat-regionalistische vertreten waren. Zum anderen wurde die Forderung nach regionaler Autonomie auch von den meisten anderen politischen Akteuren in Katalonien, insbesondere den Organisationen der Linken und der Arbeiterbewegung, vertreten - grundsätzliche Opposition dagegen war in der Region marginal. Die Artikulation dieser Forderung erfolgte nahezu ausschließlich auf gewaltfreiem Weg; Höhepunkt war anlässlich des katalanischen „Nationalfeiertages“ am 11. September 1977 eine Demonstra- 
tion für regionale Autonomie in Barcelona mit 1,5 Millionen Teilnehmern einem Viertel der Gesamtbevölkerung Kataloniens. ${ }^{35}$

Wenige Wochen später verfügte die spanische Regierung die „provisorische Wiederherstellung“ der 1939 aufgehobenen autonomen katalanischen Institutionen. In der Folge wurde - getragen zunächst von den katalanischen Abgeordneten des gesamtstaatlichen Parlaments - ein Autonomiestatut ausgearbeitet. ${ }^{36}$ Dieses war dann Grundlage für Verhandlungen mit der Zentralregierung, die auf der Streichung einiger besonders weitreichender Bestimmungen bestand. Insbesondere konnten sich die Nationalisten nicht mit ihrer Forderung nach eigener Steuerhoheit analog zum Baskenland durchsetzen, so dass die regionalen Institutionen sich aus Anteilen an den in der Region erhobenen Steuern und Zuweisungen des Zentralstaates finanzieren, im Übrigen aber ein erheblicher Teil der in Katalonien erhobenen Steuern in die Finanzierung wirtschaftlich schwächerer Landesteile fließt. Das Ende 1979 in Kraft getretene Statut sah aber weitreichende Kompetenzen u.a. in der Sozialpolitik, in der Raumordnung, der Kultur und im Bildungswesen sowie diverse exekutive Befugnisse, u.a. eine regionale Polizei (mit allerdings zunächst nur eingeschränktem Verantwortungsbereich) und eine regionale Rundfunk- und Fernsehanstalt vor. Zudem wurde das Katalanische gleichberechtigte Amtssprache.

Dieses Statut diente bis 2006 als staatsrechtliche Grundlage für die begrenzte politische Selbstbestimmung Kataloniens, wobei verschiedentlich durch zentralstaatliche Gesetze noch weitere Kompetenzen (etwa im Bildungswesen) auf die Region übertragen wurden. Zudem nutzte die größte katalanische nationalistische Partei CiU in den 1990er Jahren ihre Schlüsselstellung im spanischen Parlament, als erst eine sozialistische und dann eine konservative Minderheitsregierung auf sie als Tolerierungspartner angewiesen war, um eine verbesserte Finanzierungssituation für die Region zu erzielen, insbesondere einen erhöhten Anteil an der in Katalonien erhobenen Einkommensteuer. ${ }^{37}$

Nach dem gesamtstaatlichen Regierungswechsel 2004 wurde eine Reform des Autonomiestatus in Angriff genommen, deren Ergebnis nach schwierigen Verhandlungen zwischen regionaler und Zentralregierung ein im Juli 2006 in Kraft getretenes neues Statut war. ${ }^{38}$ Damit verband sich jedoch keine grundsätzliche

35 El País vom 13.09.1977.

36 McRoberts, K., a.a.O., $56 \mathrm{ff}$.

37 Ebd., $75 \mathrm{ff}$.

38 Morata, F.: European Integration and the Spanish "State of the Autonomies", in: ZSE, 4/4 (2006), 507528 , hier 525 . 
Veränderung des Status Kataloniens innerhalb Spaniens, sondern vielmehr eine Systematisierung und Arrondierung, in Teilen auch ein moderater Ausbau der bestehenden Kompetenzen. Am stärksten umstritten war die symbolische Frage, ob Katalonien durch das Statut als „Nation“ bezeichnet werden sollte. Das Ergebnis war eine bewusst mehrdeutige Formulierung in der Präambel, die schließlich 2010 vom spanischen Verfassungsgericht aufgrund einer Klage der konservativen Opposition für nicht rechtsverbindlich erklärt wurde. ${ }^{39}$

Mit der Einrichtung begrenzter regionaler Selbstbestimmung war man den nationalistischen Forderungen ein erhebliches Stück entgegengekommen. Entsprechend lässt sich für die Folgejahre auch eine vorübergehende Eindämmung des Konflikts konstatieren. Die 1980er und 1990er Jahre waren von Regionalregierungen der größten dem katalanisch-nationalistischen Spektrum zugerechneten Partei, der bürgerlich-liberalen CiU, geprägt. In dieser sind sowohl regionalistische Kräfte, die eine staatliche Unabhängigkeit Kataloniens ablehnen, als auch separatistische Nationalisten vertreten, wobei lange Zeit das erstgenannte Spektrum dominierte. ${ }^{40}$

Seit den 1990er Jahren gewann jedoch die explizit separatistische Linkspartei ERC an Bedeutung. Die traditionsreiche Partei konnte in den 1980er Jahren nur Ergebnisse unterhalb der $5 \%$ erzielen, konnte sich jedoch in den Regionalwahlen der 1990er Jahre deutlich steigern und erzielte in den 2000er Jahren mehrfach Ergebnisse um $15 \%{ }^{41}$ Zudem erstarkte innerhalb der CiU seit Mitte der 1990er Jahre der nationalistisch-separatistische Flügel deutlich, ${ }^{42}$ so dass sich für die 2000er Jahre eine deutliche Stärkung des katalanischen Nationalismus feststellen lässt. Dies wurde auch durch verschiedene Artikulationsformen unterstrichen, insbesondere durch die seit 2009 von der ERC und Teilen der CiU betriebenen „Volksbefragungen“ über eine staatliche Unabhängigkeit. Dabei handelte es sich um auf Gemeindeebene durchgeführte Befragungen, die zwar keinerlei rechtliche Verbindlichkeit hatten, aber mit den äußeren Zeichen einer Abstimmung versehen wurden (Wählerverzeichnis, Stimmzettel, Wahlurnen etc.) und die Unterstützung der jeweiligen nationalistisch dominierten Kommunalverwaltung genossen. Entsprechend wurden sie vor allem in ländlichen Kommunen durchge-

41 Wahlergebnisse aus Katalonien nach Generalitat de Catalunya, Departament de Governació i Relacions Institucionals: Dades electorals de totes les convocatòries, abrufbar unter http://www20.gencat.cat/.

42 Guibernau, M., a.a.O., 131. 
führt, die ohnehin schon aufgrund der Wahlergebnisse als nationalistische Hochburgen galten. ${ }^{43}$ Bemerkenswert an diesen „Befragungen“ war also weniger ihr erwartbares Ergebnis (es beteiligten sich fast nur Befürworter der Unabhängigkeit) sondern vielmehr die Tatsache, dass sich durch sie ein gewachsenes nationalistisches Selbstbewusstsein und eine stärkere Dominanz des separatistischen Flügels innerhalb der $\mathrm{CiU}$ manifestiert.

Summiert man die Stimmen der beiden wichtigsten nationalistischen Parteien CiU und ERC - lässt sich zwischen den Wahlen 1980 und 2010 ein Anstieg der Stimmanteile von $39,73 \%$ auf $45,43 \%$ feststellen. Dies und das erwähnte gewachsene nationalistische Selbstbewusstsein lassen den Schluss zu, dass von einer erfolgreichen Eindämmung oder gar Lösung des katalanischen Konflikts durch die Gewährung regionaler Autonomie nicht gesprochen werden kann, auch wenn dieser nie die Eskalationsstufen des Vergleichsfalls Baskenland erreicht hat.

\section{Flämischer Nationalismus und belgischer Föderalismus}

In den späten 1960er Jahren kam es zu einer erneuten Eskalation des belgischen Konflikts, als flämische Nationalisten die Schließung der französischsprachigen Sektion an der in Flandern gelegenen Katholischen Universität Löwen forderten - der 1425 gegründeten ältesten Hochschule der Benelux-Staaten. Nach Massendemonstrationen und teils gewaltsamen Ausschreitungen wurde die Universität schließlich 1968 geteilt und für die bisherige französischsprachige Sektion ein neuer Standort in Wallonien errichtet. ${ }^{44}$ Für die frankophonen Angehörigen der katholischen Elite des Landes war dies ein traumatisches Ereignis, dessen unmittelbare Folge die Spaltung der christdemokratischen Partei in zwei eigenständige sprachlich homogene Organisationen war. ${ }^{45}$ Die beiden anderen großen Parteien des Landes vollzogen diesen Schritt in den Folgejahren nach (die Liberalen 1972 und die Sozialisten 1978), so dass es seitdem keine relevanten gesamtbelgischen Parteien mehr gibt.

1970 wurde die erste einer Serie von Verfassungsreformen verabschiedet, durch die Belgien schrittweise von einem zentralisierten Einheitsstaat in einen Bundes-

43 El Pais vom 14. und 15.12.2009.

44 Gevers, L.: The Catholic Church and the Flemish Movement, in: Deprez, K/Vos, L (Hg.): Nationalism in Belgium. Shifting Identities, 1780-1995, Basingstoke u.a., 1998, 110-118, hier 116f.

45 Vos, L.: The Flemish National Question, in: Deprez, K/Vos, L (Hg.): Nationalism in Belgium. Shifting Identities, 1780-1995, Basingstoke u.a., 1998, 83-95, hier 93. 
staat umgewandelt wurde, der auch im Vergleich mit anderen Föderalstaaten außerordentlich dezentralisiert ist - weitere Schritte folgten 1980, 1988/89, 1993 und 2001; eine weitere Reform wird derzeit zwischen den Parteien verhandelt. Ergebnis dieser Politik ist eine eigentümlich komplexe föderale Struktur, die durch die Parallelität zweier Typen von Gebietskörperschaften auf der subnationalen Ebene gekennzeichnet ist. ${ }^{46}$

Dies liegt in der besonderen Rolle der Hauptstadt Brüssel begründet. Im Zuge des Föderalisierungsprozesses war es das Ziel der flämischen Seite, die Stadt institutionell möglichst eng an Flandern zu binden. Insbesondere die Nationalisten berufen sich dabei auf die historische Zugehörigkeit der Stadt zum flämischen Sprachgebiet und sehen in ihr die natürliche Hauptstadt Flanderns. Hingegen verwies die frankophone Seite stets auf die deutliche französischsprachige Bevölkerungsmehrheit in der Stadt und strebte eine möglichst große Eigenständigkeit Brüssels an.

Im Ergebnis ist das Land zum einen in drei Regionen eingeteilt: Flandern, Wallonien und Brüssel, wobei die Abgrenzung der 1962 festgelegten Sprachgrenze folgt und das deutsche Sprachgebiet Wallonien zugeordnet wurde. Zum anderen bestehen drei sog. Gemeinschaften: die Flämische, die Französische und die Deutschsprachige. Auch deren Einteilung folgt der amtlich festgelegten Sprachgrenze, wobei das deutsche Sprachgebiet eine eigene Gemeinschaft bildet und auf dem Gebiet Brüssels sowohl die Flämische als auch die Französische Gemeinschaft ihre Kompetenzen ausüben.

Jede dieser Körperschaften verfügt über ein eigenes Parlament und eine von diesem abhängige Regierung. Je nach Politikfeld ist sind entweder die Institutionen des Föderalstaats oder der Regionen oder der Gemeinschaften zuständig. So liegen bei der föderalen Ebene nach wie vor insbesondere die Steuerpolitik und Finanzverwaltung, die gesetzlichen Sozialversicherungen, das Justizwesen und die Verteidigung. Die Gemeinschaften verfügen über umfassende Kompetenzen im Wesentlichen in personen- und sprachbezogenen Politikfeldern, etwa dem Kultur- und Bildungswesen, der Gesundheitsversorgung und der Sozialhilfe. Hingegen liegen die Befugnisse der Regionen auf eher territorial bezogenen Aufgabenbereichen, etwa der Wirtschaftsförderung, der Infrastruktur- und Verkehrspolitik sowie der Kommunalaufsicht. Insgesamt kommt die Einrichtung der

46 Vgl. ausführlich zur institutionellen Struktur Lejeune, M.A.: Introduction au droit et aux institutions de la Belgique fédérale, 4. Aufl., Brüssel, 2004. Zu den Reformprozessen im einzelnen vgl. Mabille, X., a.a.O. 
Gemeinschaften dem flämischen Interesse entgegen, eine institutionelle Verbindung zwischen Brüssel und Flandern zu schaffen, da die Flämische Gemeinschaft auch in Brüssel öffentliche Aufgaben wahrnimmt, ${ }^{47}$ während die Stärkung der Regionen im frankophonen Interesse liegt, da durch sie der eigenständige Charakter Brüssels betont wird.

Im Ergebnis sind in Belgien inzwischen deutlich mehr öffentliche Aufgaben und Entscheidungskompetenzen auf die subnationale Ebene übertragen worden, als dies in den meisten anderen Föderalstaaten der Fall ist. Besonders augenfällig wird dies an der Tatsache, dass Änderungen der Europäischen Verträge in Belgien nicht nur vom gesamtstaatlichen Parlament ratifiziert werden müssen, sondern auch von den gesetzgebenden Körperschaften aller Regionen und Gemeinschaften. Entsprechend wird in der Literatur auch von einem „ausgehöhlten“ Zentralstaat gesprochen. ${ }^{48}$

Stellt man dem die Entwicklung des Konflikts gegenüber, so muss man zum einen feststellen, dass in den letzten Jahrzehnten die Stimmanteile flämischnationalistischer Parteien - hierunter sind insbesondere die inzwischen aufgelöste Volksunie, der rechtsextreme Vlaams Blok und seine Nachfolgepartei Vlaams Belang sowie die in jüngster Zeit dominierende Nieuw-Vlaamse Alliantie (NVA) zu fassen - stark angestiegen sind. ${ }^{49}$ Waren die nationalistischen Parteien in den 1960er Jahren noch eine Randerscheinung, kamen sie bei den jüngsten gesamtstaatlichen Parlamentswahlen im Juni 2010 auf insgesamt ca. $45 \%$ der flämischen Stimmen. ${ }^{50}$ Hinzu kommt die Tatsache, dass auch die etablierten Parteien, die wie benannt seit den 1970er Jahren als eigenständige flämische Parteien organisiert sind, ebenfalls zwar nicht explizit eine staatliche Trennung, aber doch kontinuierlich eine weitere Aufgabenverlagerung auf die subnationale Ebene verlangen, wohingegen sich zunehmender Widerstand auf frankophoner

47 Im Wesentlichen ist die Flämische Gemeinschaft auf dem Gebiet Brüssels für die niederländischsprachigen Bildungs- und Kultureinrichtungen zuständig, während die entsprechenden frankophonen Institutionen in der Stadt von der Französischen Gemeinschaft unterhalten werden. Die öffentlichen Krankenhäuser, deren Betrieb ebenfalls Sache der Gemeinschaften ist, werden von beiden Gemeinschaften gemeinsam getragen.

48 Hooghe, L.: Belgium: Hollowing the Center, in: Amoretti, U.M./Bermeo, N. (Hg.): Federalism and Territorial Cleavages, Baltimore/London, 2004, 55-92.

49 De Winter, L. u.a.: Party System(s) and Electoral Behaviour in Belgium: From Stability to Balkanisation, in: West European Politics, 29/5 (2006), 933-956.

50 SPF Intérieur: Elections 2010, http://elections2010.belgium.be/fr/. Für frühere belgische Wahlergebnisse siehe De Winter u.a., a.a.O., $935 \mathrm{ff}$. 
Seite bildet. ${ }^{51}$ Aus diesem Grund hielten die Kompromisse über die Verfassungsreformen seit 1970 im Durchschnitt keine zehn Jahre, und auch die enormen Schwierigkeiten, nach der Parlamentswahl 2010 eine Regierung zu bilden, sind dem Umstand geschuldet, dass die flämischen Parteien eine erneute Verfassungsreform mit noch weiterer Kompetenzverlagerung auf die subnationale Ebene zur Bedingung für eine Koalition machten. Ein stabiles ,,institutionelles Gleichgewicht" zwischen Zentrum und Gebietseinheiten konnte sich bislang nicht herausbilden.

\section{Fazit: kanalisierte, aber nicht gelöste Konflikte}

Vergleicht man die drei geschilderten Fälle, so lässt sich eine Reihe von Gemeinsamkeiten feststellen. Zunächst bestand jeweils ein politischer Konflikt insofern, als relevante Akteure im Namen einer baskischen, katalanischen bzw. flämischen Nation die Schaffung eines unabhängigen Staates forderten. Dem wurde jeweils durch eine Dezentralisierung der Staatsorganisation begegnet, also die Einrichtung entsprechender regionaler Gebietskörperschaften und deren Ausstattung mit substanziellen politischen Befugnissen. Dies geschah im Baskenland und in Flandern in besonders weitreichender Form, doch auch in Katalonien sind die autonomen regionalen Kompetenzen beachtlich.

Dennoch führten diese Maßnahmen in keinem der drei Fälle zu einer dauerhaften Lösung des Konflikts. Weder rückten die nationalistischen Akteure von ihren Forderungen nach Unabhängigkeit ab, noch ging die Unterstützung ihrer Positionen in der Bevölkerung zurück. Vielmehr konnten die baskischen Nationalisten seit Einrichtung der regionalen Autonomie ihren Stimmanteil konstant halten; in Katalonien und Flandern stiegen die Stimmenanteile der nationalistischen Parteien seit Einrichtung der regionalen Selbstregierung sogar signifikant an.

Dagegen wurde - ebenfalls in allen drei Fällen - eine Mäßigung der Konfliktaustragung erreicht. Zwar machten nur im baskischen Fall die Nationalisten von terroristischer Gewalt Gebrauch, doch auch in Katalonien und in Flandern kam es vor Einrichtung der autonomen regionalen Institutionen zu Massendemonstrationen, Akten des zivilen Ungehorsams und in Flandern auch zu teils gewaltsamen Ausschreitungen. Diese Phänomene sind in den beiden letztgenannten Fällen heute weitgehend überwunden, auch im Baskenland konnte das Ausmaß der Gewalt stark reduziert werden. Insofern lässt sich festhalten, dass durch die Poli- 
tik der Dezentralisierung der Staatsorganisation immerhin eine institutionelle Arena geschaffen wurde, die eine geregelte Form der Konfliktaustragung möglich macht. Die Nationalitätenkonflikte im Baskenland, in Katalonien und in Flandern wurden durch die Dezentralisierungspolitiken zwar nicht gelöst, aber doch insofern kanalisiert, als sie von den Straßen in die Parlamente wanderten. 